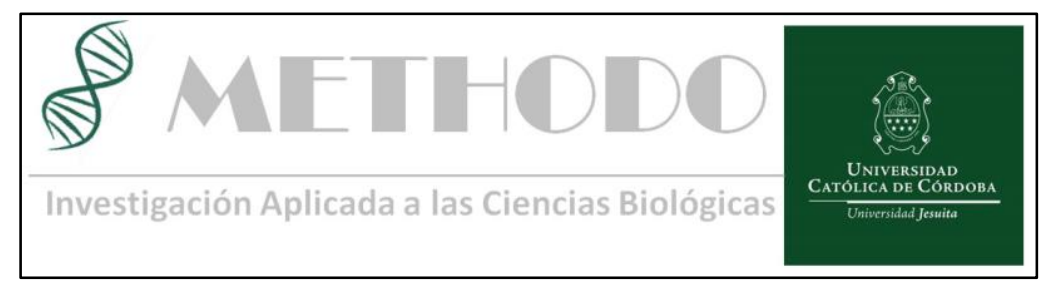

ARTICULO ORIGINAL Methodo 2017;2(2):55-58 DOI: $10.22529 / \mathrm{me} .2017 .2(2) 05$

Recibido 03 Dic 2016 | Aceptado 17 Abr 2017 | Publicado 30 Jn 2017

\title{
Bevacizumab intravítreo como tratamiento de maculopatías miópicas neovasculares *
}

\section{Intravitreal bevacizumab for neovascular myopic maculopathy}

${ }^{*}$ Resumen deTrabajo Presentado en las XIII Jornadas de Investigación de la Clínica Universitaria Reina Fabiola- Diciembre de 2016

Dana Martinez M1, Gonzalez-Castellanos E1, Barros-Centeno MF1, Correa L1, Guantay CD1, Serra HM2, Urrets-Zavalia JA1.

\section{RESUMEN}

Introducción. La miopía patológica es una causa importante de pérdida de visión irreversible y es la cuarta a novena causa más frecuente de ceguera en el mundo. Es también conocida como miopía alta, degenerativa o maligna; condición en la que los individuos tienen una longitud axial superior a 25,5 - 26,5mm, y/o un error de refracción de por lo menos -5.0 dioptrías, acompañado por cambios patológicos.

La neovascularización coroidea (NVC) asociada a miopía patológica puede resultar en la pérdida significativa de la visión y/o la ceguera. La NVC suele ser subfoveal y es una importante complicación, desarrollándose en aproximadamente $5-10 \%$ de ojos con miopía patológica.
De manera similar que en otras enfermedades maculares asociadas a NVC, se ha encontrado un aumento del nivel del factor de crecimiento de endotelial (VEGF) en NVC miópicas, y por lo tanto, la terapia anti-VEGF sería útil.

Desde la introducción en oftalmología de agentes anti factor de crecimiento de endotelial (anti-VEGF), el tratamiento anti-angiogénico con antiVEGF intravítreo se ha convertido en el tratamiento de primera línea para la NVC miópica.

El bevacizumab es un anticuerpo monoclonal humano, anti factor de crecimiento endotelial (anti-VEGF), que inhibe la proliferación de nuevas células endoteliales produciendo un bloqueo de la fosforilación de las uniones estrechas (tight junctions) de las mismas. Este mecanismo produciría 
Dana Martinez M, Gonzalez-Castellanos E, Barros-Centeno MF, Correa L, Guantay CD, Serra HM, Urrets-Zavalia JA. Bevacizumab intravítreo como tratamiento de maculopatías miópicas neovasculares una mejoría anatómica-funcional en los pacientes e impediría una de las complicaciones más importantes de esta patología como lo es la neovascularización.

Objetivo. Evaluar la eficacia de bevacizumab intravítreo (Avastin ${ }^{\mathrm{T}}{ }^{\mathrm{T}}$ ) como tratamiento de la neovascularización coroidea (NVC) en miopías patológicas.

Pacientes y métodos. Se evaluaron retrospectivamente 22 pacientes con diagnóstico de maculopatía miópica neovascular tratados mediante inyección intravítrea de bevacizumab, con un seguimiento mínimo de 12 meses. La agudeza visual se evaluó mediante tabla de Snellen y se convirtió en unidades LogMAR. El espesor macular se evaluó mediante tomografía de coherencia óptica (OCT). Las variables cuantitativas se analizaron mediante medidas de tendencia central, dispersión y forma. Los cambios en la agudeza visual se calcularon utilizando la prueba de Wilcoxon para variables apareadas y con la prueba de Mann Whitneypara comparar variables independientes. Las diferencias entre variables continuas con distribución normal y de muestras independientes fueron calculadas mediante la prueba $\mathrm{T}$ de Student.

Resultado. Se estudiaron 22 pacientes con diagnóstico de maculopatía miópica neovascular, cuya edad promedio fue de 59,68 (DE 11,75; rango $34,00-85,00)$, de los cuales 7 $(31,8 \%)$ fueron hombres y $15(68,2 \%)$ fueron mujeres. El tiempo de seguimiento fue de 12 meses. El tiempo promedio transcurrido entre el comienzo de los síntomas y el inicio del tratamiento fue 38,68 (DE 34,63) días. El 68,2\% (15) de los pacientes consultaron por disminución brusca de la agudeza visual del ojo afectado y $31,8 \%$ (7) consultaron por metamorfopsias. Todos los pacientes presentaron miopía patológica (> 5.0 dioptrías).

La cantidad total de inyecciones durante el seguimiento tuvo una media de 4,27 (DE 1.86; Rango 2,00 - 9,00), con un máximo de 9 inyecciones y un mínimo de 2 inyecciones. Durante los primeros 6 meses se realizaron la mayor parte de las inyecciones con una media de 3,36 (DE 1,22; Rango 1,00 $6,00)$.

La mediana de la AV al momento del tratamiento fue de 1,00 (P25-75=0,401,00). Al analizar la totalidad de los pacientes se encontró que existe una diferencia significativa al comparar las agudezas visuales previas al tratamiento y a los 12 meses de tratamiento $(p=<0.0001)$.. La mejoría 
Dana Martinez M, Gonzalez-Castellanos E, Barros-Centeno MF, Correa L, Guantay CD, Serra HM, Urrets-Zavalia JA. Bevacizumab intravítreo como tratamiento de maculopatías miópicas neovasculares franca de la AV se observó entre el

primer mes (mediana $=1.00 \mathrm{RIQ}=0,6$ )

y los 3 meses de tratamiento

(mediana $=0,60 \mathrm{RIQ}=0,6)(\mathrm{p}=0,0002)$,

mientras que no hubo diferencias

significativas en la variación de la AV

más allá de los 3 meses de

seguimiento $(p=0,09)$.

Al estudiar los espesores maculares

antes del tratamiento, encontramos una mediana de $290(\mathrm{RIQ}=105)$; mientras

que a los 12 meses de seguimiento fue de 269,50 ( $R I Q=91)$. Teniendo en cuenta el total de los pacientes estudiados, no hubo diferencias significativas en el análisis del espesor macular medido por OCT antes y después del tratamiento $(p=0,8812)$.

Conclusiones. El bevacizumab fue eficaz en el tratamiento de la maculopatía miópica, si bien no se encontraron diferencias significativas en la variación del espesor macular. En nuestra serie no hubo complicaciones oculares ni sistémicas vinculadas al tratamiento.

\section{ABSTRACT}

Background: Pathological myopia is observed in about $2 \%$ of the general population. Submacular choroidal neovascularization is a leading cause of severe visual loss and blindness in eyes with pathological myopia, affecting $4-11 \%$ of those eyes.

Purpose: Our aim is to evaluate the efficacy and safety of intravitreal bevacizumab in the treatment of neovascular myopic maculopathy (NMM).

Patients and methods: 22 nonpreviously treated eyes of 22 consecutive patients with NMM. were treated with monthly intravitreal injections of bevacizumab and followed up for 12 months. Changes in BCVA and central macular thickness were evaluated at 12 months of follow-up. Snellen best-corrected visual acuity (BCVA) was converted into LogMAR units. Mean central macular thickness was obtained by means of spectral domain optical coherence tomography (SP-OCT). Quantitative variables were analyzed with central tendency, dispersion and shape. Changes in BCVA were calculated with Wilcoxon test in paired variables. Mann Whitney test was used to compare independent variables. Differences between continuous variables with normal distribution and independent samples were calculated with the Student T test. Main outcome measures: Changes in BCVA and central macular thickness at12 months of follow-up.

Results: Mean age was 54.45 (SD $12.30 ; r=28.00-79.00) ; 7$ patients (31.8\%) were male and 15 (68.2\%) female. Mean spherical equivalent refractive error was $-10.89 \pm 4.13(r=$ 
Dana Martinez M, Gonzalez-Castellanos E, Barros-Centeno MF, Correa L, Guantay CD, Serra HM, Urrets-Zavalia JA. Bevacizumab intravítreo como tratamiento de maculopatías miópicas neovasculares 7.00 to $-21,00$ ) Mean time elapsed stable during follow-up.

between initial symptoms and the beginning of treatment was 38.68 (SD34.63) days. Patients received a mean of 4.27 (SD 1.86; r=2.00 to 9.00) injections. Most injections were performed during the first 6 months of treatment (mean 3.36 months; SD 1.22; $r=1.00$ to 6.00 ). Median BCVA at baseline was 1.00 (P25-75=0.40-1.00) and at 12 months 0.45 (P25-75=0.30$0.70)(p<0.0001)$. Significant visual improvement was observed between the first (median=1.00, IQR=0.6) and the third month of treatment (median=0.60, IQR=0.6) $(p=0.0002)$, with no further significant improvement $(p=0.09)$. No ocular or systemic side effects attributable to treatment were observed. When comparing patients 55 years old or younger with those older, and between both genders, all individuals improved, although not significantly (Mann Whitney for age $\mathrm{P}=0.1765$; gender $\mathrm{P}=3454$ ). No significant improvement in macular thickness was observed (pretreatment median thickness 290 microns, IQR=105; month 12 post-treatment median thickness 269.5 microns, IQR= 91) $(P=0.8812)$.

\section{Conclusions: Bevacizumab was}

effective and safe in our series of myopic patients with neovascular maculopathy, and visual gain remained 